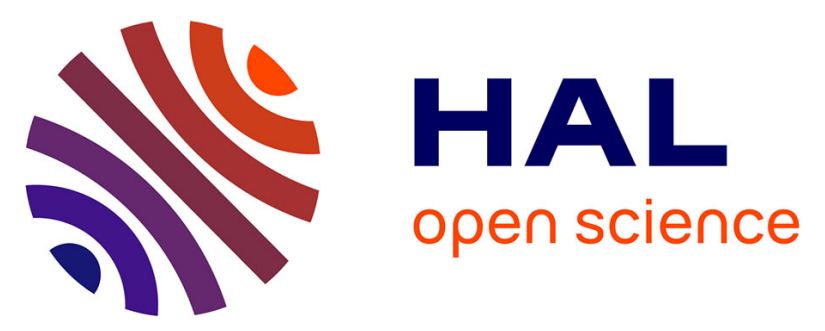

\title{
Type II fatty acid synthesis pathway and cyclopropane ring formation are dispensable during Enterococcus faecalis systemic infection
}

Constantin Hays, Clara Lambert, Sophie Brinster, Gilles Lamberet, Laurence Du Merle, Karine Gloux, Alexandra Gruss, Claire Poyart, Agnès Fouet

\section{To cite this version:}

Constantin Hays, Clara Lambert, Sophie Brinster, Gilles Lamberet, Laurence Du Merle, et al.. Type II fatty acid synthesis pathway and cyclopropane ring formation are dispensable during Enterococcus faecalis systemic infection. Journal of Bacteriology, 2021, 203 (20), pp.e0022121. 10.1128/JB.0022121. hal-03408405

\section{HAL Id: hal-03408405 https://hal.science/hal-03408405}

Submitted on 29 Oct 2021

HAL is a multi-disciplinary open access archive for the deposit and dissemination of scientific research documents, whether they are published or not. The documents may come from teaching and research institutions in France or abroad, or from public or private research centers.
L'archive ouverte pluridisciplinaire $\mathbf{H A L}$, est destinée au dépôt et à la diffusion de documents scientifiques de niveau recherche, publiés ou non, émanant des établissements d'enseignement et de recherche français ou étrangers, des laboratoires publics ou privés. 
1 Type II fatty acid synthesis pathway and cyclopropane ring formation are dispensable during

2 Enterococcus faecalis systemic infection

3

4 Constantin Hays $^{\mathrm{a}, \mathrm{b}, \mathrm{c}^{*}}$, Clara Lambert ${ }^{\mathrm{a}}$, Sophie Brinster ${ }^{\mathrm{a}}$, Gilles Lamberet ${ }^{\mathrm{d}} \uparrow$, Laurence du Merle ${ }^{\mathrm{e}, \mathrm{f}}$,

5 Karine Gloux ${ }^{\mathrm{d}}$, Alexandra Gruss ${ }^{\mathrm{d}}$, Claire Poyart ${ }^{\mathrm{a}, \mathrm{b}, \mathrm{c}, \#^{\dagger}}$, Agnes Fouet $\mathrm{t}^{\mathrm{a}, \mathrm{b}} \#^{\dagger}$

6

7 anniversité de Paris, Institut Cochin, INSERM, CNRS, F-75014 Paris, France

$8 \quad{ }^{b}$ Centre National de Référence des Streptocoques, Paris, France

9 'Hôpitaux Universitaires Paris Centre, Site Cochin, Assistance Publique Hôpitaux de Paris, Paris,

10 France.

11 dMICALIS, UMR1319, Institut National de Recherche pour l'Agriculture, l'Alimentation et

12 l'Environnement, Domaine de Vilvert, 78352 Jouy en Josas, France.

13 'Institut Pasteur, Unité de Biologie des Bactéries Pathogènes à Gram Positif, Paris, France

$14{ }^{\mathrm{f} C N R S}$ ERL3526, Paris, France.

16 Running head: E. faecalis FASII and $c f a$ are dispensable in infection

18 \#Address correspondence to Agnes Fouet agnes.fouet@inserm.fr, Claire Poyart

19 claire.poyart@inserm.fr.

$20{ }^{*}$ Present address, Laboratoire d'Immunologie et Histocompatibilité, Saint-Louis Hospital, Paris,

21 France.

$22 \dagger$ Deceased

$23{ }^{+}$These authors contributed equally 


\section{Abstract}

25 Enterococcus faecalis, a multi-antibiotic-resistant Gram-positive bacterium, has emerged as a

26 serious nosocomial pathogen. Here, we used a genetic approach to characterize the strategies used

27 by E. faecalis to fulfill its requirements for endogenous fatty acid (FA) synthesis in vitro and in

28 vivo. The FA synthesis (FASII) pathway is encoded by two operons and two monocistronic genes.

29 Expression of all these genes is repressed by exogenous FAs, which are incorporated in the $E$.

30 faecalis membrane and modify its composition. Deletion of nine genes of the 12-gene operon

31 abolished growth in a FA-free medium. Addition of serum, which is lipid-rich, restored growth.

32 Interestingly, the E. faecalis membrane contains cyclic fatty acids that modify membrane

33 properties, but are unavailable in host serum. The $c f a$ gene that encodes the cyclopropanation

34 process, is located in a locus independent of the FASII genes. Its deletion did not alter growth under

35 the conditions tested, but yielded bacteria devoid of cyclic FAs. No differences were observed

36 between mice infected with wild-type, or FASII or cyclopropanation mutant strains, in terms of

37 bacterial loads in blood, liver, spleen or kidneys. We conclude that in E. faecalis, neither FASII

38 nor cyclopropanation enzymes are suitable antibiotic targets.

\section{Importance}

41 Membrane lipid homeostasis is crucial for bacterial physiology, adaptation, and virulence. Fatty

42 acids are constituents of the phospholipids that are essential membrane components. Most bacteria

43 incorporate exogenous fatty acids into their membranes. Enterococcus faecalis has emerged as a

44 serious nosocomial pathogen, which is responsible for urinary tract infections, bacteremia and

45 endocarditis, and is intrinsically resistant to numerous antibiotics. E. faecalis synthesizes saturated

46 and unsaturated fatty acids, but also cyclic fatty acids that are not found in the human host. We 
47 characterized mutant strains deficient in fatty acid synthesis and modification using genetic,

48 biochemical, and in vivo approaches. We conclude that neither the fatty acid synthesis pathway nor

49 the cyclopropanation enzyme are suitable targets for E. faecalis antibiotic development. 


\section{INTRODUCTION}

51 Bacterial fatty acids (FAs) are usually synthesized by the type II Fatty Acid Synthesis

52 (FASII) pathway. FAs constitute the two hydrophobic tails which, together with a hydrophilic head,

53 comprise phospholipids (PL) that are essential components of cell membranes. Enterococcus

54 faecalis synthesizes both saturated and unsaturated fatty acids (SFA and UFA, respectively), as do

55 numerous streptococci (1-3). Additionally, E. faecalis and numerous other bacteria encode a

56 cyclopropane fatty acid synthase (Cfa), which catalyzes the transfer of a methyl group from S-

57 adenosyl-L-methionine to the double bond of a lipid chain, thereby forming a cyclopropane ring

58 (4). Recent studies uncovered that E. faecalis also synthesizes trans-UFA, further expanding the variety of fatty acids produced by this bacterium (5). Both cyclopropane and trans fatty acids are more rigid than their non-modified substrates, and contribute to E. faecalis membrane homeostasis

61 in starvation and stress conditions (6-8).

FASII pathway defects prevent bacterial growth in vitro in FA-non-supplemented media;

63 this suggested that FASII enzymes may be essential for bacterial viability and therefore feasible

64 targets for antibiotic design (9-15). However, most bacteria incorporate exogenous FA into their

65 membranes. FA abundance in host compartments and in serum, led us to hypothesize, and then

66 prove that FA incorporation can bypass FASII-targeted antibiotics in the neonatal pathogen

67 Streptococcus agalactiae, as well as other Streptococcaceae (1). Nevertheless, antibiotic bypass

68 may vary according to whether bacteria can survive on exclusively exogenous FAs; for example,

69 Mycobacterium tuberculosis remains sensitive to the anti-FASII isoniazid because of its complex

70 lipid requirement (16). In contrast, Staphylococcus aureus, which synthesizes FAs not produced

71 by humans, can nevertheless bypass FASII inhibitors with host-derived fatty acids (17-19).

Enterococcus faecalis is a Gram-positive bacterium that inhabits the gastrointestinal tract

73 of humans and animals (20) and has long been associated with food fermentations. E. faecalis has 
74 emerged as a serious nosocomial pathogen, and is the second most frequently isolated Gram-

75 positive pathogen isolated in hospitals after S. aureus $(21,22)$. E. faecalis is responsible for urinary

76 tract infections, bacteremia and endocarditis (23). It is intrinsically resistant to many antibiotics,

77 and its acquisition of antibiotic resistance genes has made it a public health concern worldwide

78 (23). This situation prompts the search for new antibiotic targets, notably within the FASII pathway

$79(24,25)$.

80 Whether targeting FASII pathway or cyclopropanation enzymes could lead to treatment of

81 E. faecalis systemic infection is not resolved. E. faecalis was shown to use environmental fatty

82 acids (26). In the presence of $15 \%$ human serum, E. faecalis membrane comprises about $10 \%$

83 vaccenic acid (VA; C18:1 $\Delta 11$ ), an FA not present in serum (26). Thus, the E. faecalis FASII

84 pathway is likely active even in the presence of exogenous FAs. The E. faecalis FASII pathway is

85 encoded by the twelve gene cluster, $f a b T-a c c A$, whose genes are present in numerous Gram-

86 positive species, and a set of three genes, $f a b O$ - $f a b N$ and the divergent $f a b I$ gene, encoding

87 enzymes involved in UFA and SFA synthesis, respectively $(14,27)$. Finally, $c f a$ encodes the

88 cyclopropanation reaction. In one study, deletion of $f a b N$ resulted in E. faecalis UFA auxotrophy

89 (28). In E. faecalis, expression of FASII genes is controlled by FabT, the transcriptional repressor.

90 An auxiliary acyl-carrier-protein carrying long chain FAs binds to FabT, and facilitates repression

91 of a consensus sequence that regulates FASII gene expression $(14,29,30)$.

92 Although E. faecalis incorporates FA from its environment, its FA synthesis requirement

93 in an FA-rich environment cannot be deduced $(31,32)$. In this study we characterized the repressive

94 effect of serum on expression of FASII and FA-related genes. We also tested whether FASII

95 pathway and/or cyclopropanation functions are possible antibiotic targets in a murine model of $E$.

96 faecalis systemic infection. 
Bacterial strains, media and culture conditions. E. faecalis V583 vancomycin resistant

strain was isolated from a bloodstream infection and sequenced. It was used for the in silico analysis. VE14089, a plasmid-cured derivative of the V583 strain, was the wild type (WT) reference strain used for the construction of mutant and back to the wild type (BWT) strains (33, 34). Two mutants were constructed, Ef $\Delta$ FASII and Ef $\Delta$ cfa corresponding to the in-frame deletion of nine genes from $f a b K$ to $a c c A(7863 \mathrm{bp})$ and of $c f a(1176 \mathrm{bp})$, respectively; the isogenic BWT strains were obtained, BWT-FASII and BWT-cfa, respectively. Escherichia coli TG1 (Invitrogen) was used for cloning experiments. E. faecalis was cultured in Todd-Hewitt (TH) broth or agar 106 (Difco Laboratories, Detroit, MI) or in RPMI $^{+}$(RPMI-1640; Life technologies, Gibco) 107 supplemented with amino acids 1X (Gibco®, Life Technologies), vitamins 1X (Sigma Aldrich), glucose $1 \%$ and Hepes $0.1 \mathrm{mM}$ (Gibco ${ }^{\circledR}$, Life Technologies). E. coli was cultured in TrypticaseSoy (TS) medium (Difco Laboratories, Detroit, MI). When needed, antibiotics were used at the

110 following concentrations: for $E$. coli, ticarcillin, $100 \mu \mathrm{g} / \mathrm{ml}$, and erythromycin, $160 \mu \mathrm{g} / \mathrm{ml}$; for $E$.

111 faecalis, erythromycin, $50 \mu \mathrm{g} / \mathrm{ml}$. Cerulenin (Sigma) was added at $8 \mu \mathrm{g} / \mathrm{ml}$ final concentration.

In silico analysis. The AGTTTGATAATCAAATT sequence was used to seek potential

113 FabT binding sites in the regulatory regions or within the FASII genes (27).

General DNA techniques. E. coli plasmid DNA was prepared by rapid alkaline lysis using

115 the QIAprep Spin Miniprep kit (Qiagen). Genomic DNA from E. faecalis was prepared using the 116 DNeasy Blood and Tissue Kit (Qiagen) with pretreatment for Gram-positive bacteria as 117 recommended by the manufacturer. PCR was carried out with the AmpliTaq Gold polymerase as 118 described by the manufacturer (Applied Biosystems). The molecular weight standard used in 119 agarose gels is the $1 \mathrm{~kb}$-plus DNA ladder (Life Technologies). Amplification products were purified 120 on QIAquick PCR purification kit (Qiagen). PCR products and plasmids used were sequenced with 
121 an ABI 310 automated DNA sequencer, using the ABI PRISM dyes terminator cycle sequencing

122 kit (Applied Biosystem). To determine the genetic and transcriptional organization of VE14089

123 FASII genes, primer pairs were designed to flank neighboring FASII genes. Primers are listed in

124 Table S1.

125 Strain construction. The primers used for the construction of the in-frame Ef $\Delta$ FASII and

126 Ef $\Delta$ cfa mutant strains are listed in Table S1. Primers used are based on the published genome

127 sequence of E. faecalis V583 (GenBank accession number NC004668.1) (33). The $\Delta$ FASII

128 deletion was constructed by using splicing-by-overlap extension PCR as described (35). The

129 corresponding PCR fragment was cloned into the thermosensitive shuttle plasmid pG1. The $\Delta c f a$

130 (ef0203, NCBI reference sequence NC_004668.1) deletion was constructed using In-Fusion PCR

131 Cloning Kit (Clontech), cloning the two PCR-amplified fragments into pG1 previously linearized

132 in a one-step reaction following manufacturer's recommendations. The resulting intermediate

133 plasmids, pG1 $\triangle$ FASII and pG1 $\Delta c f a$, were checked by sequencing the inserts. Electroporation of

134 VE14089 and allelic exchange were performed as described with minor modifications $(35,36)$. For

135 the Ef $\Delta$ FASII construction Tween $800.1 \%$ (Sigma), a rich source of FA containing $70 \%$ oleic acid

136 (OA; C18:1 $\Delta 9)$, was added in all steps of the experiment after the electroporation. The in-frame

137 deletion mutants and BWT strains were confirmed by PCR and sequence analysis.

138 Growth curves. E. faecalis strains were inoculated into $\mathrm{RPMI}^{+}$supplemented with Tween

$13980(0.1 \%)$ and cultured overnight at $37^{\circ} \mathrm{C}$. Overnight cultures were washed twice in PBS and sub-

140 cultured (1:100) in $\mathrm{RPMI}^{+}$and in $\mathrm{RPMI}^{+}$supplemented with $40 \%$ human serum. Growth was

141 determined by measuring absorbance at $600 \mathrm{~nm}\left(\mathrm{OD}_{600}\right)$. Experiments were repeated three times.

142 RNA extraction and RT-PCR. RNA was extracted from bacteria by the phenol

143 chloroform technique as described (37). RNA $(2-5 \mu \mathrm{g})$ was treated with DNAse I (Promega) 
144 according to manufacturer's recommendations. RNA was quantified by absorbance at 260 and 280

$145 \mathrm{~nm}$. RNA purity and integrity were controlled and RNA was stored at $-80^{\circ} \mathrm{C}$ until use.

146 Reverse-transcription was carried out using the Superscript II kit (Invitrogen) and Random

147 Hexamer Primers (Fermentas), according to manufacturers' instructions. PCRs were carried out on

148 cDNA, as well as on gDNA and RNA as positive and negative controls, using primer pairs flanking

149 neighboring FASII genes (Table S1). PCR Amplicons were examined on a 1\% agarose gel.

150 Real-time quantitative PCRs. Overnight VE14089 RPMI $^{+}$cultures were then subcultured

151 (1:100) in $\mathrm{RPMI}^{+}$with or without addition of $40 \%$ human serum to mid-log phase $\left(\mathrm{OD}_{600}=0.3\right)$.

152 RNAs were extracted and cDNA samples were diluted to $50 \mathrm{ng} / \mu \mathrm{l}$. qPCR was performed using the

153 LightCycler (Roche) and the SYBR Green PCR kits (Applied Biosystems) using primers listed in

154 Table S1. Each assay was performed in duplicate, with two independently prepared total RNA

155 samples. The relative quantification in gene expression was determined by the $2^{-\Delta \Delta C t}$ method, using $156 r p o \mathrm{~B}$ as the reference gene (38).

157 Fatty acid analysis. Strains were inoculated into RPMI ${ }^{+}$supplemented with Tween 80 $158(0.1 \%)$ and grown overnight at $37^{\circ} \mathrm{C}$. Cells were washed three times in $\mathrm{RPMI}^{+}$, diluted 100 -fold in 159 fresh $\mathrm{RPMI}^{+}$cultures with or without $40 \%$ human serum. Cells were harvested after $14 \mathrm{~h}$ of growth 160 (stationary phase) in static conditions at $37^{\circ} \mathrm{C}$, and washed once in $\mathrm{NaCl} 0.9 \%$ containing $0.01 \%$ 161 Triton $\mathrm{X}-100$ and twice in $\mathrm{NaCl} 0.9 \%$. Cell pellets were stored at $-20^{\circ} \mathrm{C}$ until analysis. The FA 162 composition of serum used for experiments was also determined. Whole cell esterified fatty acid 163 determinations were done on an AutoSystem XL Gas Chromatograph (Perkin-Elmer) equipped 164 with a DB-Wax (Agilent, XXX; for all analyses except those shown in Fig. 3D) or ZB-Wax 165 capillary column (30 m x $0.25 \mathrm{~mm} \times 0.25 \mathrm{~mm}$; Phenomenex, France; for Fig. 3D) as described (1, 166 17). Bacterial fatty acid composition of each sample was analyzed in three independent 167 experiments. 
Ethics statement. All animal experiments described in this study were conducted in

169 accordance with guidelines of Paris Descartes University, in compliance with the European animal 170 welfare regulation (http://ec.europa.eu/environment/chemicals/lab_animals/home_en.html) and

171 were approved by the University Paris Descartes animal care and use committee and by the 172 Ministère de l'Education Nationale, l'Enseignement Supérieur et la Recherche 173 (2015032714098562_v1, APAFIS \#390).

$174 \quad$ Intravenous infection of mice. All in vivo infections in this study were performed with 6175 week-old female BALB/c mice (Charles River, L'Arbresle cedex, France). Bacterial suspensions 176 used for intravenous (i.v.) infection were prepared as follows. Overnight broth cultures of mutant 177 and isogenic BWT strains, grown in TH supplemented with $0.1 \%$ Tween 80, were diluted 1:100 178 into $50 \mathrm{ml}$ of $\mathrm{TH}$-Tween 80 medium and grown until $\mathrm{OD}_{600}=0.6$. The bacterial suspensions were 179 washed twice in $\mathrm{NaCl} 0.9 \%$ and resuspended in $\mathrm{NaCl} 0.9 \%$ to obtain from $6 \times 10^{8}$ to $8 \times 10^{8}$ colony 180 forming units $(\mathrm{CFU}) / \mathrm{ml}$ final concentration in $500 \mu \mathrm{l}$ for intravenous (iv) injections. Mice were 181 injected with a 28-gauge, 0.5 -in. needle in the tail vein.

182 Dissemination assay and organ analysis. Randomized groups of 9-21 mice or of 6 mice,

183 BWT-FASII and Ef $\triangle$ FASII, and BWT-cfa and and Ef $\Delta$ cfa, respectively, were infected and mice 184 were sacrificed by cervical dislocation at days 1,7 and 14 or day 7, post infection (pi); blood, liver, 185 spleen and kidneys were harvested. All organs were weighed prior to further experimentation. 186 Liver, spleen and kidneys were homogenized in $0.9 \% \mathrm{NaCl}$. Serial dilutions were immediately 187 plated on TH agar plates containing $0.1 \%$ Tween80 (TH-Tween agar). CFU were enumerated after $18824 \mathrm{~h}$ incubation at $37^{\circ} \mathrm{C}$.

189 Statistical analysis. Statistical analyses were performed using a 2-way ANOVA and a 190 Bonferroni post-test or a Mann-Whitney test (PRISM 8). A $p$ value $\leq 0.05$ was considered 191 statistically significant; $* \mathrm{p}<0.05, * * \mathrm{p}<0.01, * * * \mathrm{p}<0.005, * * * * \mathrm{p}<0.0001$ 
FASII pathway genetic organization and regulation of expression. In E. faecalis, a total of 15 genes belonging to the FASII pathway were previously identified $(14,27,39)$. However,

195 a $16^{\text {th }}$ gene, ef1773 (NCBI reference sequence for the protein, WP_010706667.1), named fabG, is 196 present in the annotated V583 genome. This gene is conserved among E. faecalis isolates. As two 197 genes were assigned the same annotation we suggest renaming ef2881, which belongs to the 12 198 gene cluster, as $f a b G a$, and $e f 1773$, fabGb. FabGa and FabGb proteins share $32 \%$ identity and $65 \%$ 199 similarity. Physiological and enzymatic properties of the two FabG homologues remain unknown 200 and we hypothesized that they may catalyze the same 3-oxoacyl-ACP reduction step but with 201 different substrate preferences or conditions of expression. In silico analysis using the E. faecalis 202 FabT consensus sequence indicated the presence of a putative FabT binding site, 203 AATTTGAAACAAAACG (palindromic nucleotides in bold), 10 bp downstream of the $f a b G b$ 204 translation start codon (Fig. 1A) (39). This further suggests that FabGb is involved in FA synthesis.

205 Genetic organization of the 12 gene cluster was initially defined in silico on the basis of an upstream 206 FabT consensus binding sequence (27). Depending on the species, an organization in two or three 207 operons was suggested $(14,27,39)$. In E. faecalis, FabT consensus binding sequences are present 208 upstream of $f a b T$ and $f a b K$ (Fig. 1A), suggesting a two-operon organization. Also, the UFA 209 synthesis genes, $f a b O$ and $f a b N$, may constitute another operon $(14,27,39)$. This organization was 210 tested by overlapping RT-PCR mapping (Fig. 1A,1B). Results show that the 12-gene cluster is 211 organized as a single operon, which ends before ef2874. A transcription reinitiation site may be 212 linked to the FabT consensus binding sequence upstream of fabK. Furthermore, respectively 213 intense and faint bands were obtained for $f a b O-f a b N$ and $f a b N-p y r D-1$ (=ef0285), suggesting that $214 f a b O$ and $f a b N$ constitute an operon and that there may be some read-through downstream of $f a b N$. 
215 Altogether our data show that E. faecalis FASII genes are organized in two operons and two 216 monocistronic transcriptional units (Fig. 1A,1B).

217 The FASII pathway can be strongly repressed by exogenous fatty acids, such that

218 phospholipid synthesis relies on exogenous FA substrates $(1,40)$. Human serum is rich in lipids 219 comprising long acyl-chain fatty acids such as 18:1 (15\%) and 16:0 (25\%), which can act as FabT 220 corepressors (29). To our knowledge, the regulation of E. faecalis FASII gene expression elicited 221 by addition of FAs has not been studied. To address this question, and after checking that there was 222 no growth difference in the presence or absence of added human serum (data not shown), we used 223 qRT-PCR to analyze the effect of $40 \%$ human serum on E. faecalis FASII gene expression (Fig. $2241 \mathrm{~A}, 1 \mathrm{C})$. Serum addition resulted in decreased expression of all FASII genes varying from 2.5- to 225 14-fold depending on the gene. Interestingly, a putative FabT binding site was found close to the 226 most strongly repressed genes, $f a b G b, f a b O$ and $f a b K$. This suggests that a FabT-controlled 227 transcription initiation site or a transcription repression site exists upstream of these genes. 228 Altogether, our data show that the 12 gene operon (from $f a b T$ to $a c c A$ ), as defined above, is strongly 229 repressed in the presence of a physiological concentration of serum.

E. faecalis FASII pathway and cyclopropanation are dispensable for growth in the

presence of serum. Supplementation with serum or unsaturated FA overcomes growth inhibition mediated by cerulenin, a FASII inhibitor $(1,17,41)$. We confirmed these results with the WT strain 233 VE14089 (data not shown). The consequences of a non-functional FASII pathway were then 234 examined by constructing Ef $\triangle$ FASII, in which 9 genes of the 12-gene operon, from fabK to acc $A$ 235 (ef2883 to ef2875) were deleted. The genes that were removed encode products implicated in the 236 initiation and elongation modules (Fig. 1A). Growth of the WT, EfAFASII mutant and back-to237 the-wild-type (BWT-FASII) (see Materials and Methods) strains was first compared in RPMI ${ }^{+}$ 238 (Fig. 2A). The WT and the BWT-FASII strains behaved similarly. In contrast, Ef $\Delta$ FASII growth 
239 was essentially arrested in $\mathrm{RPMI}^{+}$; slight observed growth is likely due to residual Tween 80 used

240 in precultures. These results confirm that an active FASII pathway is required for growth in FA-

241 free medium. Addition of $40 \%$ human serum to growth medium led to equivalent growth of all

242 strains (Fig. 2A), indicating that serum is sufficient to fully restore growth of the Ef $\Delta$ FASII mutant

243 strain. Altogether, these results indicate that the exogenous FAs present in serum are necessary and

244 sufficient to restore growth when E. faecalis FASII pathway is abolished, either via the addition of

245 a FASII inhibitor (1) or by deletion of FASII genes.

246 The role of cyclopropanation on E. faecalis growth in the presence of exogenous FAs

247 remained an open question. E. faecalis produces cyclopropane FAs, which are produced from both

248 endogenous and exogenous substrates, presumably to mediate membrane fluidity adjustment by

249 modifying UFA (8). A cfa in-frame deletion mutant was constructed to assess the role of

250 cyclopropane FA synthesis in E. faecalis growth in medium without or with host serum. The mutant

251 strain grew like the WT and BWT-cfa strains in both media, indicating that the presence or absence

252 of cyclic FA does not impact $E$. faecalis growth in vitro (Fig 2B).

E. faecalis WT and Ef $\triangle$ FASII strains show identical FA composition upon growth in

254 serum. Strong repression of E. faecalis FASII genes in the presence of human serum correlates

255 with incorporation of FAs, as previously observed in the same culture condition (26). We

256 determined FA compositions of WT VE14089 and BWT derivative (BWT-FASII) grown in the

257 absence or presence of $40 \%$ serum (Table S2, S3, Fig. 3A). BWT-FASII profiles were similar to

258 those of the WT; the slight differences probably arose from growth variations that could alter the

259 efficiency of conversion between the UFA and cyclo forms. As previously described, FA of cells

260 grown in FA-free medium $\left(\mathrm{RPMI}^{+}\right)$comprised mainly palmitic acid (16:0; PA) (40\%), vaccenic

261 acid $\left(18: 1 \Delta^{11}\right.$; VA) $(20 \%)$ and cyclopropanated-VA form $\left(\mathrm{C} 19 \Delta^{11}\right)(20 \%)$ (Fig. 3A). In contrast, 
262 membrane FA composition of bacteria grown in serum resembled profiles of serum alone (Table

263 S3, Fig. 3A) $(14,26,31)$. Thus, the proportion of endogenously produced $18: 1 \Delta^{11}$ dropped to $1.7 \%$,

264 and that of serum-provided oleic acid $\left(18: 1 \Delta^{9}\right.$; OA) rose to $13.5 \%$. These latter percentages are

265 different from previously reported ones $(26,31)$; this could be due to the use here of $40 \%$ rather

266 than 15\% serum. Thus, VE14089, like other E. faecalis isolates, incorporates serum-supplied

267 exogeneous FA (6). Another notable change in the presence of serum was a decrease in the amount

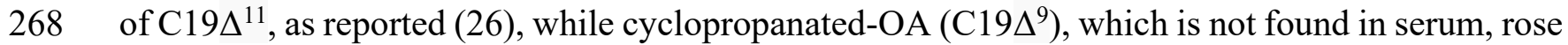

269 to $15 \%$, indicating that $E$. faecalis CFA synthase cyclizes both $18: 1 \Delta^{9}$ and $18: 1 \Delta^{11}$. This indicates

270 that E. faecalis CFA synthase is active on different substrates which is in agreement with the fact

271 that bacterial CFAs display lax requirements for positioning of the cis double bond, except for cis

272 double-bond positions at FA extremities, or trans double bonds, which are generally not converted 273 (4).

We also established the FA content of the Ef $\Delta$ FASII mutant strain grown in $40 \%$ human

275 serum (Fig. 3B). The FA profiles of the Ef $\triangle$ FASII and BWT-FASII strains were similar (Fig. 3B).

276 Both strains similarly incorporated serum FAs and generated $\mathrm{C} 19 \Delta^{9}$. These data demonstrate that

277 FASII activity has no impact on membrane composition in the presence of human serum.

\section{E. faecalis cyclopropane fatty acid synthase impacts the cellular FA composition .}

279 To characterize the impact of the $c f a$ deletion on E. faecalis membrane FA composition, we 280 compared FA profiles of Ef $\Delta c f a$, BWT-cfa, and WT strains grown in FA-free medium and in the presence of human serum (Fig. 3C, 3D, Tables S2, S4). The BWT-cfa, profiles were near identical

282 to those of the WT (Tables S2, S4). Compared to the BWT-cfa control strain in RPMI ${ }^{+}$the Ef $\Delta c f a$ 283 mutant strain was devoid of $C 19 \Delta^{11}$ and was enriched in 18:1 $\Delta^{11}$ (Fig. 3C). In serum-supplemented 284 medium, the Ef $\Delta c f a$ strain was devoid of $\mathrm{C} 19 \Delta^{9}$ and enriched in $18: 1 \Delta^{9}$ (Fig. 3D). These data 285 confirm that the Ef $\Delta c f a$ strain is defective for cyclopropanation of unsaturated fatty acids, mainly 
18:1 $1 \Delta^{11}$ in $\mathrm{RPMI}^{+}$and $18: 1 \Delta^{9}$ in the presence of serum. This implies that, in vivo, the fatty acid

composition of $c f a$ defective strains may be different from those of wild-type strains and this could have physiological consequences.

E. faecalis septicemic infection is independent of both the FASII pathway and fatty acid cyclopropanation. The WT, Ef $\triangle$ FASII and BWT-FASII strains grew similarly and displayed the same FA composition when grown in the presence of human serum. During infection however, E. faecalis infects organs whose FA contents are variable $(42,43)$. We therefore asked whether exogenous FAs in the host may compensate the loss of the active FASII pathway in vivo.

We first followed the infection process, looking at the physiological consequences of intravenous infection with the BWT-FASII strain. CFU counts and organ weights were examined 1, 7, and 14 days post infection (pi) (Fig. 4A, 4B). Throughout the course of the experiment, the

CFUs in blood were below the detection threshold (data not shown). The CFU counts in the liver diminished 31-fold, at $14 \mathrm{~d}$ pi, with no change in the weight of livers throughout the experiment.

299 In the spleen, the bacterial levels strongly decreased over a 2-week period, from approximately $10^{4}$ $300 \mathrm{CFU} 24 \mathrm{~h} \mathrm{pi}$, to $66 \%$ spleens being sterile at the end of the experiment. However, d14-spleens 301 weighed twice as much as d1 spleens (Fig. 4B). Finally, kidney weights increased from d7 to d14 302 (Fig. 4B). This correlated, albeit with a time laps, to the tendency in the CFU counts increase at d7 303 in BWT-FASII-infected mice (15-fold, respectively) and is in agreement with E. faecalis tropism 304 for the kidneys $(31,32)$.

We then assessed the consequences of the FASII gene deletion by comparing the BWT-

306 FASII- and Ef $\Delta$ FASII-infected mice. Bacterial counts in liver, spleen and kidneys, as well as organ 307 weights, were similar for BWT-FASII- and Ef $\Delta$ FASII-infected mice at all-time points (Fig. 4A, 308 4B). This demonstrates that, as in other species, targeting the FASII pathway would be ineffective 309 for the treatment of E. faecalis septicemic infection (1). 
311 model. Cfa is reportedly more expressed in stationary phase (44). We reasoned that differences, if

312 any, between Ef $\Delta \mathrm{cfa}$ and BWT-cfa would be more pronounced after CFUs reached their highest

313 level in the wild-type strain (here BWT) -infected mice, i.e., at d7. No differences were observed

314 in terms of bacterial loads in the different organs between Ef $\Delta$ cfa- and BWT-cfa-infected mice

315 (Fig. 4C). This indicates that cyclopropanation activity does not confer an advantage to E. faecalis

316 during systemic infection in this model.

317 In conclusion, our data demonstrate that targeting the FASII pathway or the 318 cyclopropanation process would be ineffective for the treatment of E. faecalis septicemic infection. 


\section{ACKNOWLEDGMENTS}

320 We fondly acknowledge our colleague G. Lamberet, who passed away Dec 23, 2019. We

321 thank A. Tazi for helpful discussions.

322 This work was supported by funding from the Agence Nationale de la Recherche (ANR-

323 13001038), the Fondation pour la recherche Médicale (DBF20161136769) and from INSERM,

324 CNRS and Université de Paris. 


\section{REFERENCES}

326 1. Brinster S, Lamberet G, Staels B, Trieu-Cuot P, Gruss A, Poyart C. 2009. Type II fatty acid 327 synthesis is not a suitable antibiotic target for Gram-positive pathogens. Nature 458:83-86.

328 2. Altabe S, Lopez P, de Mendoza D. 2007. Isolation and characterization of unsaturated fatty 329 acid auxotrophs of Streptococcus pneumoniae and Streptococcus mutans. J Bacteriol $330 \quad 189: 8139-8144$.

331 3. Montgomerie JZ, Kalmanson GM, Guze LB. 1973. Fatty acid composition of L-forms of 332 Streptococcus faecalis cultured at different osmolalities. J Bacteriol 115:73-75.

333 4. Grogan DW, Cronan JE. 1997. Cyclopropane ring formation in membrane lipids of bacteria. 334 Microbiol Mol Biol Rev MMBR 61:429-441.

335 5. Kondakova T, Kumar S, Cronan JE. 2019. A novel synthesis of trans-unsaturated fatty acids 336 by the Gram-positive commensal bacterium Enterococcus faecalis FA2-2. Chem Phys Lipids $337 \quad 222: 23-35$.

338 6. Zhang Y-M, Rock CO. 2008. Membrane lipid homeostasis in bacteria. Nat Rev Microbiol $339 \quad 6: 222-233$.

340 7. Parsons JB, Rock CO. 2013. Bacterial lipids: metabolism and membrane homeostasis. Prog $341 \quad$ Lipid Res 52:249-276.

342 8. Poger D, Mark AE. 2015. A ring to rule them all: the effect of cyclopropane fatty acids on the 343 fluidity of lipid bilayers. J Phys Chem B 119:5487-5495.

344 9. Heath RJ, White SW, Rock CO. 2001. Lipid biosynthesis as a target for antibacterial agents. $345 \quad$ Prog Lipid Res 40:467-497.

346 10. Parsons JB, Rock CO. 2011. Is bacterial fatty acid synthesis a valid target for antibacterial 347 drug discovery? Curr Opin Microbiol 14:544-549. 
11. Zhang Y-M, White SW, Rock CO. 2006. Inhibiting bacterial fatty acid synthesis. J Biol Chem 281:17541-17544.

12. Heath RJ, White SW, Rock CO. 2002. Inhibitors of fatty acid synthesis as antimicrobial chemotherapeutics. Appl Microbiol Biotechnol 58:695-703.

13. Lu H, Tonge PJ. 2008. Inhibitors of FabI, an enzyme drug target in the bacterial fatty acid biosynthesis pathway. Acc Chem Res 41:11-20.

14. Zhu L, Bi H, Ma J, Hu Z, Zhang W, Cronan JE, Wang H. 2013. The two functional enoylacyl carrier protein reductases of Enterococcus faecalis do not mediate triclosan resistance.

15. Parsons JB, Frank MW, Rosch JW, Rock CO. 2013. Staphylococcus aureus fatty acid

16. Bhatt A, Molle V, Besra GS, Jacobs WR, Kremer L. 2007. The Mycobacterium tuberculosis auxotrophs do not proliferate in mice. Antimicrob Agents Chemother 57:5729-5732. mBio 4:e00613-13-e00613-13. FAS-II condensing enzymes: their role in mycolic acid biosynthesis, acid-fastness, pathogenesis and in future drug development: the mycobacterial FAS-II condensing enzymes. Mol Microbiol 64:1442-1454.

17. Kénanian G, Morvan C, Weckel A, Pathania A, Anba-Mondoloni J, Halpern D, Gaillard M, Solgadi A, Dupont L, Henry C, Poyart C, Fouet A, Lamberet G, Gloux K, Gruss A. 2019. Permissive fatty acid incorporation promotes staphylococcal adaptation to FASII antibiotics in host environments. Cell Rep 29:3974-3982.e4.

18. Gloux K, Guillemet M, Soler C, Morvan C, Halpern D, Pourcel C, Vu Thien H, Lamberet G, Gruss A. 2017. Clinical relevance of type II fatty acid synthesis bypass in Staphylococcus aureus. Antimicrob Agents Chemother 61:e02515-16, e2515-16.

19. Morvan C, Halpern D, Kénanian G, Hays C, Anba-Mondoloni J, Brinster S, Kennedy S, 
enable emergence of infectious Staphylococcus aureus resistant to FASII-targeted $373 \quad$ antimicrobials. Nat Commun 7.

374 20. Fisher K, Phillips C. 2009. The ecology, epidemiology and virulence of Enterococcus. $375 \quad$ Microbiology 155:1749-1757.

376 21. Karchmer AW. 2000. Nosocomial bloodstream infections: organisms, risk factors, and 377 implications. Clin Infect Dis 31:S139-S143.

378 22. Wisplinghoff H, Bischoff T, Tallent SM, Seifert H, Wenzel RP, Edmond MB. 2004. 379 Nosocomial bloodstream infections in US hospitals: analysis of 24,179 cases from a 380 prospective nationwide surveillance study. Clin Infect Dis 39:309-317.

381 23. Arias CA, Murray BE. 2012. The rise of the Enterococcus: beyond vancomycin resistance. $382 \quad$ Nat Rev Microbiol 10:266-278.

383 24. Feng Z, Chakraborty D, Dewell SB, Reddy BVB, Brady SF. 2012. Environmental DNA384 encoded antibiotics fasamycins A and B inhibit FabF in type II fatty acid biosynthesis. J Am $385 \quad$ Chem Soc 134:2981-2987.

386 25. Gerusz V. 2010. Recent advances in the inhibition of bacterial fatty acid biosynthesis. Annu $387 \quad$ Rep Med Chem 45:295-311.

388 26. Saito HE, Harp JR, Fozo EM. 2014. Incorporation of exogenous fatty acids protects 389 Enterococcus faecalis from membrane-damaging agents. Appl Environ Microbiol 80:6527$390 \quad 6538$.

391 27. Eckhardt TH, Skotnicka D, Kok J, Kuipers OP. 2013. Transcriptional regulation of fatty acid 392 biosynthesis in Lactococcus lactis. J Bacteriol 195:1081-1089.

393 28. Diederich A-K, Duda KA, Romero-Saavedra F, Engel R, Holst O, Huebner J. 2016. Deletion 394 of fabN in Enterococcus faecalis results in unsaturated fatty acid auxotrophy and decreased 395 release of inflammatory cytokines. Innate Immun 22:284-293. 
29. Jerga A, Rock CO. 2009. Acyl-Acyl carrier protein regulates transcription of fatty acid biosynthetic genes via the FabT repressor in Streptococcus pneumoniae. J Biol Chem 284:15364-15368.

30. Zhu L, Zou Q, Cao X, Cronan JE. 2019. Enterococcus faecalis encodes an atypical auxiliary acyl carrier protein required for efficient regulation of fatty acid synthesis by exogenous fatty acids 10:16.

31. Harp JR, Saito HE, Bourdon AK, Reyes J, Arias CA, Campagna SR, Fozo EM. 2016. Exogenous fatty acids protect Enterococcus faecalis from daptomycin-induced membrane stress independently of the response regulator LiaR. Appl Environ Microbiol 82:4410-4420.

32. Kau AL, Martin SM, Lyon W, Hayes E, Caparon MG, Hultgren SJ. 2005. Enterococcus faecalis tropism for the kidneys in the urinary tract of C57BL/6J mice. Infect Immun 73:2461-2468.

33. Paulsen IT, Banerjei L, Myers GSA, Nelson KE, Seshadri R, Read TD, Fouts DE, Eisen JA, Gill SR, Heidelberg JF, Tettelin H, Dodson RJ, Umayam L, Brinkac L, Beanan M, Daugherty S, DeBoy RT, Durkin S, Kolonay J, Madupu R, Nelson W, Vamathevan J, Tran B, Upton J, Hansen T, Shetty J, Khouri H, Utterback T, Radune D, Ketchum KA, Dougherty BA, Fraser CM. 2003. Role of mobile DNA in the evolution of vancomycin-resistant Enterococcus faecalis. Science 299:2071-2074.

34. Rigottier-Gois L, Alberti A, Houel A, Taly J-F, Palcy P, Manson J, Pinto D, Matos RC, Carrilero L, Montero N, Tariq M, Karsens H, Repp C, Kropec A, Budin-Verneuil A, Benachour A, Sauvageot N, Bizzini A, Gilmore MS, Bessières P, Kok J, Huebner J, Lopes F, Gonzalez-Zorn B, Hartke A, Serror P. 2011. Large-scale screening of a targeted Enterococcus faecalis mutant library identifies envelope fitness factors. PLoS ONE 6:e29023. 
419 35. Biswas I, Gruss A, Ehrlich SD, Maguin E. 1993. High-efficiency gene inactivation and 420 replacement system for Gram-positive bacteria. J Bacteriol 175:3628-3635.

421 36. Cruz-Rodz AL, Gilmore MS. 1990. High efficiency introduction of plasmid DNA into glycine 422 treated Enterococcus faecalis by electroporation. Mol Gen Genet MGG 224:152-154.

423 37. Lamy M-C, Zouine M, Fert J, Vergassola M, Couve E, Pellegrini E, Glaser P, Kunst F, 424 Msadek T, Trieu-Cuot P, Poyart C. 2004. CovS/CovR of group B Streptococcus: a two425 component global regulatory system involved in virulence: The $\operatorname{CovS} / \operatorname{CovR}$ regulatory 426 system of Streptococcus agalactiae. Mol Microbiol 54:1250-1268.

427 38. Livak KJ, Schmittgen TD. 2001. Analysis of relative gene expression data using real-time 428 quantitative PCR and the 2(-Delta Delta C(T)) Method. Methods San Diego Calif 25:402$429 \quad 408$.

430 39. Lu Y-J, White SW, Rock CO. 2005. Domain swapping between Enterococcus faecalis FabN and FabZ proteins localizes the structural determinants for isomerase activity. $\mathrm{J}$ Biol Chem 280:30342-30348.

40. Yao J, Rock CO. 2017. Exogenous fatty acid metabolism in bacteria. Biochimie 141:30-39.

41. Saito HE, Harp JR, Fozo EM. 2018. Enterococcus faecalis responds to individual exogenous 435 fatty acids independently of their degree of saturation or chain length. Appl Environ Microbiol 84.

42. Cunnane SC, McAdoo KR, Prohaska JR. 1986. Lipid and fatty acid composition of organs 438 from copper-deficient mice. J Nutr 116:1248-1256.

43. Gentry-Weeks C, Estay M, Loui C, Baker D. 2003. Intravenous mouse infection model for 440 studying the pathology of Enterococcus faecalis infections. Infect Immun 71:1434-1441. 
441 44. To TMH, Grandvalet C, Tourdot-Marechal R. 2011. Cyclopropanation of membrane 442 unsaturated fatty acids is not essential to the acid stress response of Lactococcus lactis subsp. $443 \quad$ cremoris. Appl Environ Microbiol 77:3327-3334. 


\section{FIGURE LEGENDS}

446 Figure 1 Genetic organization of $E$. faecalis FASII pathway and impact of exogenous FAs on

447 E. faecalis FASII gene expression. (A) Schematic representation of E. faecalis VE14089 FASII

448 pathway genes. Gene positions with names below are represented with open arrows. Two genes in

449 the locus, $p y r D-1$ (ef0285), and ef2874, are unrelated to FASII. Above, asterisks indicate putative

450 FabT binding sites; below, transcripts predicted by PCR reactions, straight arrows; transcripts

451 identified by predicted FabT binding sequence and expression levels, dotted arrow. (B) Agarose

452 gel of the RT-PCR amplification products on cDNA using primer pairs from neighboring genes.

453 The order is the same as that in Fig. 1A above; lane: 1, fabI-fabO; 2; fabO-fabN; 3 fabN-ef0285; 4, 454 fabT-fabH; 5, fabH-acpP; 6, acpP-fabK; 7, fabK-fabD; 8, fabD-fabGa; 9, fabGa-fabF; 10, fabF$455 a c c B ; 11, a c c B-f a b Z ; 12, f a b Z-a c c C ; 13, a c c C-a c c D ; 14, a c c D-a c c A ; 15, a c c A-e f 2874$. MW, 456 molecular weight standard. (C) FASII gene expression fold change in E. faecalis grown in RPMI 457 medium in the absence or the presence of $40 \%$ human serum. The means \pm SD of three independent 458 experiments are represented.

460 Figure 2. Impact of FASII pathway mutations on $\boldsymbol{E}$. faecalis growth. (A) Growth curves of 461 VE14089 (WT) (O), EfDFASII ( $\mathbf{\Delta})$ and BWT-FASII ( $\mathbf{\square})$ strains in RPMI ${ }^{+}$in the absence (empty 462 symbols) or presence (closed symbols) of 40\% human serum. (B) Growth curves of VE14089 (O), $463 \operatorname{Ef} \Delta c f a(\mathbf{\Delta})$ and BWT-cfa $(\boldsymbol{\square})$ strains in $\mathrm{RPMI}^{+}$in the absence (empty symbols) or presence (closed 464 symbols) of $40 \%$ human serum. Growth curves shown are representative of at least 3 independent 465 experiments. 
with serum. E. faecalis was grown in $\mathrm{RPMI}^{+}$or in $\mathrm{RPMI}^{+}$containing $40 \%$ human serum to stationary phase. FA composition was compared to assess the roles of medium FA content and genetic background. The means of the percentages of each FA found in E. faecalis, BWT or mutant 471 strains, grown to stationary phase in the presence or absence of $40 \%$ serum of three independent 472 experiments are presented. (see also Table S2, S3, S4). (A) BWT-FASII grown in the absence or 473 presence of serum. (B). BWT-FASII and Ef $\triangle$ FASII strains grown in 40\% serum. (C) BWT-cfa and 474 Ef $\Delta$ cfa strains grown in RPMI ${ }^{+}$. (D) BWT-cfa and Ef $\Delta$ cfa strains grown in $40 \%$ human serum. 475 Only major fatty acids ( $>2 \%$ of total in at least one profile) are shown. Green stars, fatty acids 476 derived from serum. Green arrows: cyclopropanation in the BWT strains of unsaturated fatty acids, 477 dark green, produced by E. faecalis (mainly $18: 1 \Delta^{11}$ ) or, light green, incorporated from serum 478 (mainly 18:1 $1 \Delta^{9}$ ). Columns: dark green BWT-FASII; dark blue, Ef $\Delta$ FASII; light green, BWT-cfa; 479 light blue, Ef $\Delta \mathrm{cfa}$; hatched, strains grown in $\mathrm{RPMI}^{+}$; empty, strains in $\mathrm{RPMI}^{+}$supplemented with $480 \quad 40 \%$ human serum.

Figure 4. EfAFASII and BWT-FASII infection in a mouse septicemic model. (A) CFUs shown 483 by scattergraph plots at days 1, 7 and 14 pi of bacteria recovered from BWT-FASII and Ef $\Delta$ FASII 484 infected mice. Nine to 20 mice were intravenously infected with $6.10^{8}$ to $8.10^{8}$ E. faecalis BWT485 FASII (closed squares) or Ef $\Delta$ FASII (open squares). (B) Organ weights shown by scattergraph 486 plots at days 1, 7 and 14 pi of 3 to 9 mice infected by Ef $\Delta$ FASII or BWT-FASII. Symbols are as 487 in 'A'. (C) CFUs shown by scattergraph plots at day 7 pi of viable bacteria recovered Ef $\Delta$ cfa and 488 BWT-cfa infected mice. Six mice were intravenously infected with 6-8.10 E. faecalis BWT-cfa 489 (closed circles) or Ef $\Delta$ cfa (open circles) strains. Upper panels, liver; middle panels, spleen; lower 
490 panels, kidneys. Each symbol represents the number of CFUs per organ of one infected mouse or

491 the weight of one organ. Median ranges are represented. Statistical analysis, A, B, 2-way ANOVA

492 and a Bonferroni post-test; C, Mann-Whitney test. ${ }^{*} \mathrm{p}<0.05 ;{ }^{* *} \mathrm{p}<0.01,{ }^{* * *} \mathrm{p}<0.005{ }^{* * * *}$ $493 \mathrm{p}<0.0001$. 
A

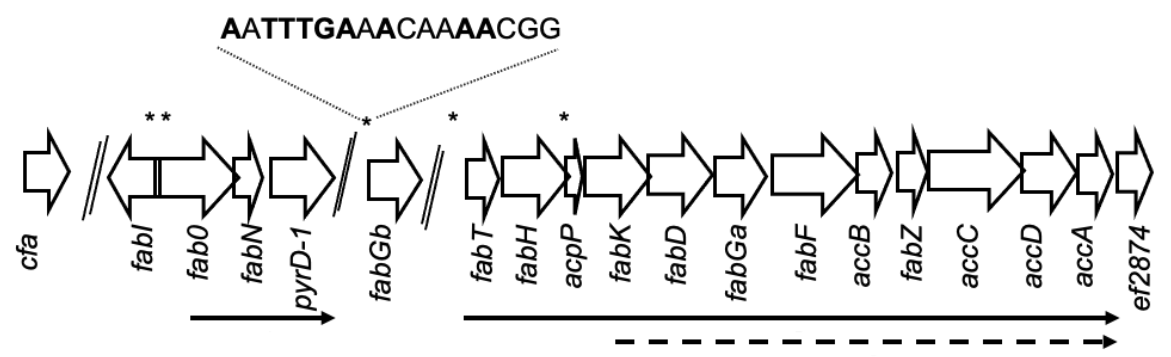

B

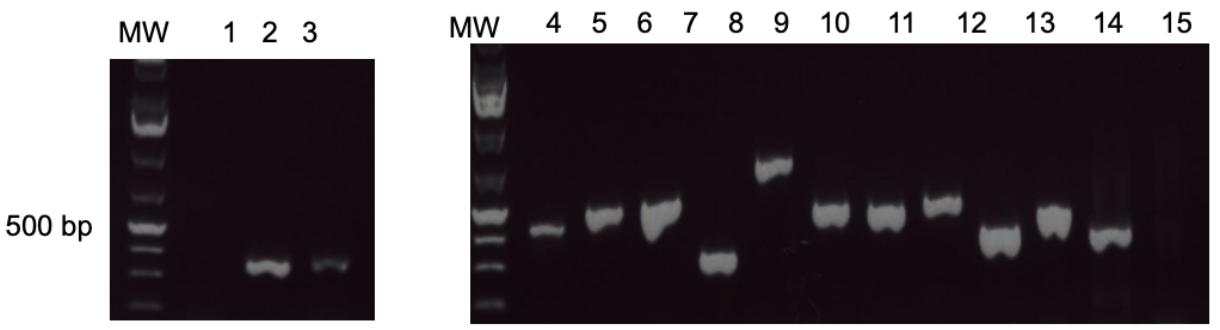

C

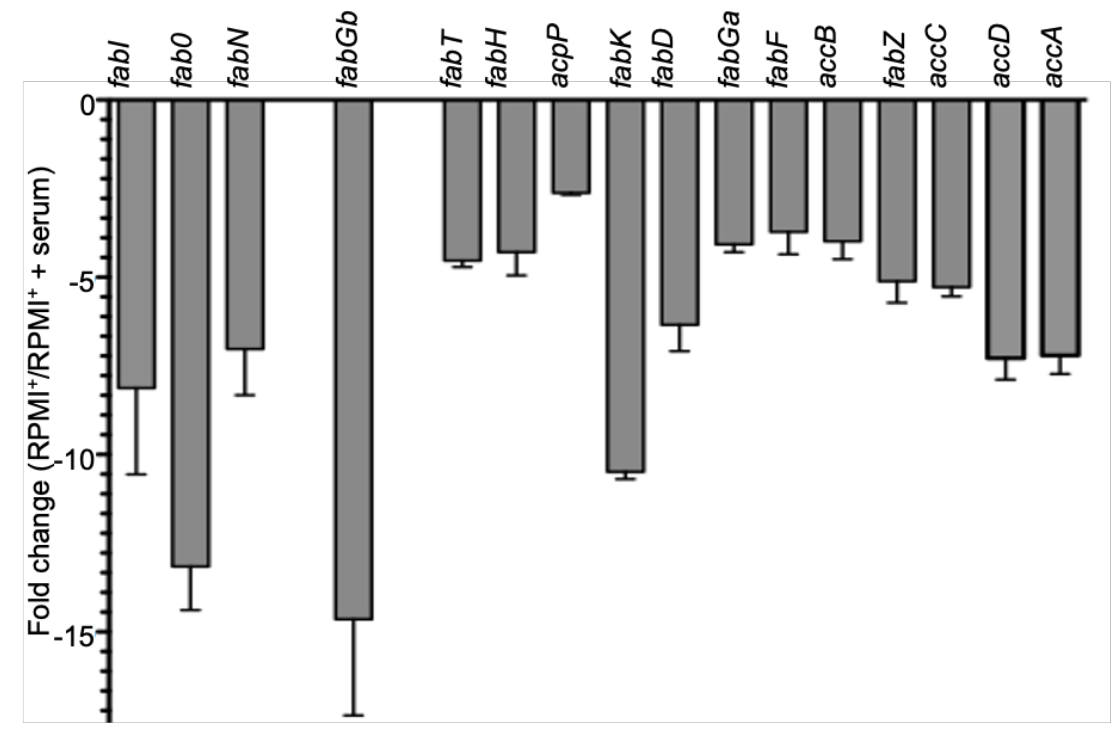

495 Figure 1 Genetic organization of $\boldsymbol{E}$. faecalis FASII pathway and impact of exogenous FAs on pathway genes. Gene positions with names below are represented with open arrows. Two genes in the locus, pyrD-1 (ef0285), and ef2874, are unrelated to FASII. Above, asterisks indicate putative FabT binding sites; below, transcripts predicted by PCR reactions, straight arrows; transcripts identified by predicted FabT binding sequence and expression levels, dotted arrow. (B) Agarose gel of the RT-PCR amplification products on cDNA using primer pairs from neighboring genes. The order is the same as that in Fig. 1A above; lane: 1, fabI-fabO; 2; fabO-fabN; 3 fabN-ef0285; 4 , fabT-fabH; 5, fabH-acpP; 6, acpP-fabK; 7, fabK-fabD; 8, fabD-fabGa; 9, fabGa-fabF; 10, fabF$a c c B ; 11, a c c B-f a b Z ; 12, f a b Z-a c c C ; 13$, accC-accD; 14, accD-accA; 15, accA-ef2874. MW, molecular weight standard. (C) FASII gene expression fold change in $E$. faecalis grown in $\mathrm{RPMI}^{+}$ 
A

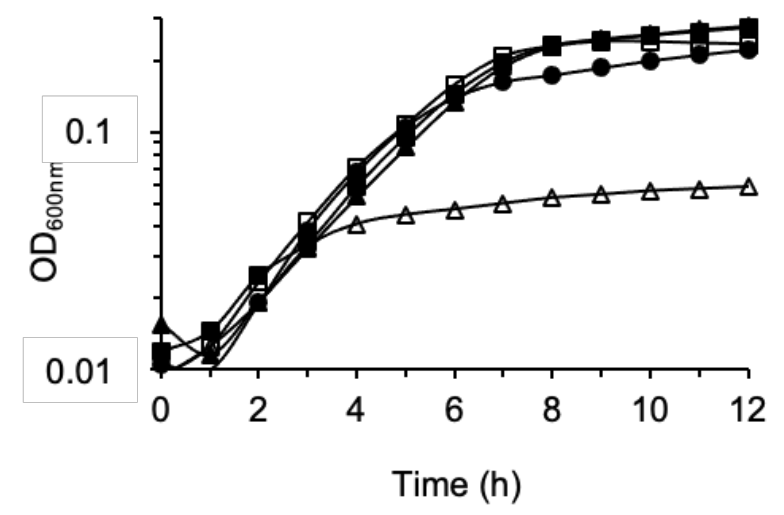

B

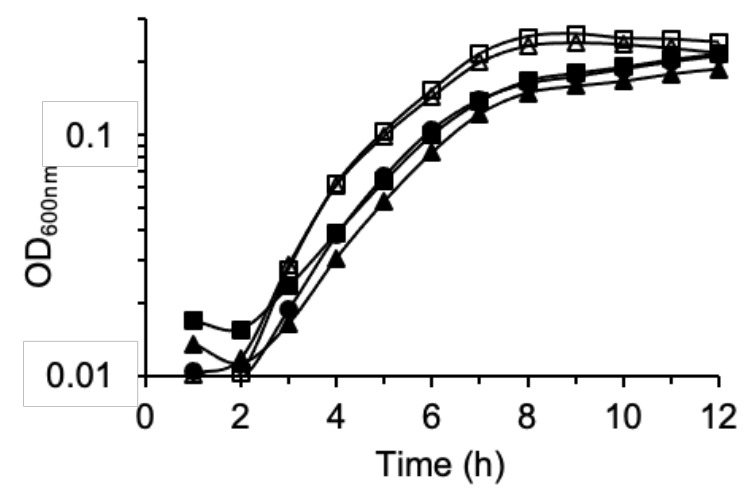

509 Figure 2. Impact of FASII pathway mutations on $\boldsymbol{E}$. faecalis growth. (A) Growth curves of 510 VE14089 (WT) (O), EfAFASII ( $\mathbf{\Delta})$ and BWT-FASII (ם) strains in $\mathrm{RPMI}^{+}$in the absence (empty 511 symbols) or presence (closed symbols) of 40\% human serum. (B) Growth curves of VE14089 (O), 512 Ef $\Delta c f a(\mathbf{\Delta})$ and BWT-cfa $(\boldsymbol{\square})$ strains in $\mathrm{RPMI}^{+}$in the absence (empty symbols) or presence (closed 513 symbols) of $40 \%$ human serum. Growth curves shown are representative of at least 3 independent 514 experiments. 
A

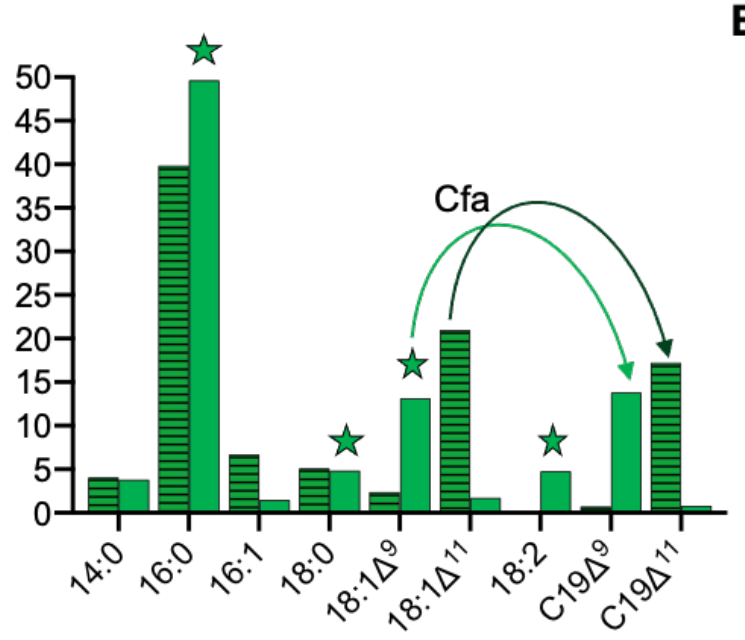

C

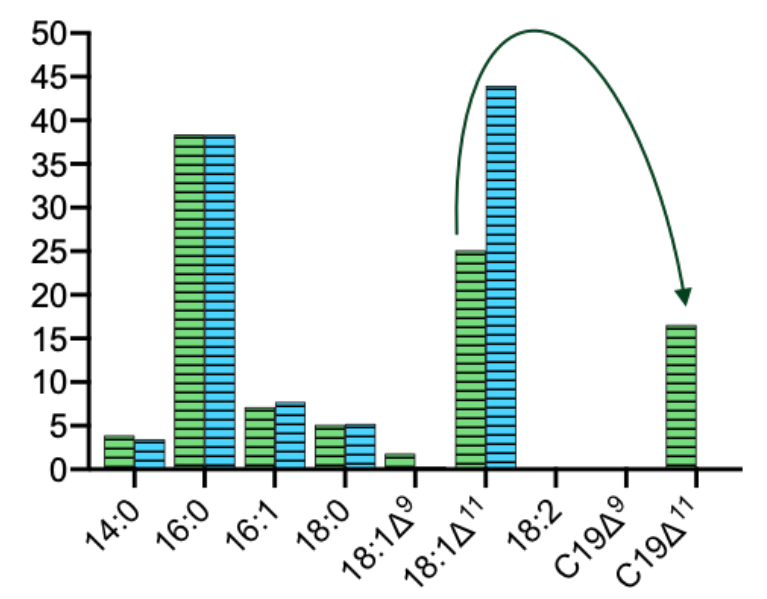
$40 \%$ human serum.
B

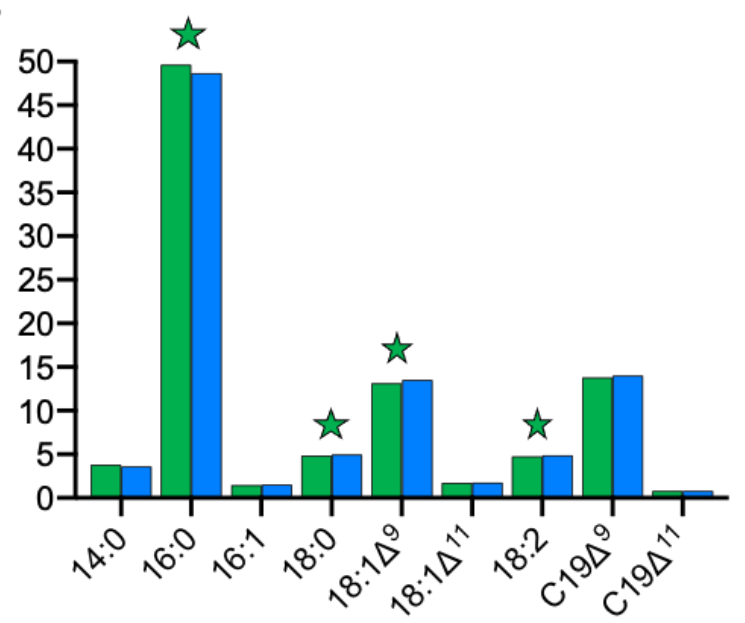

D

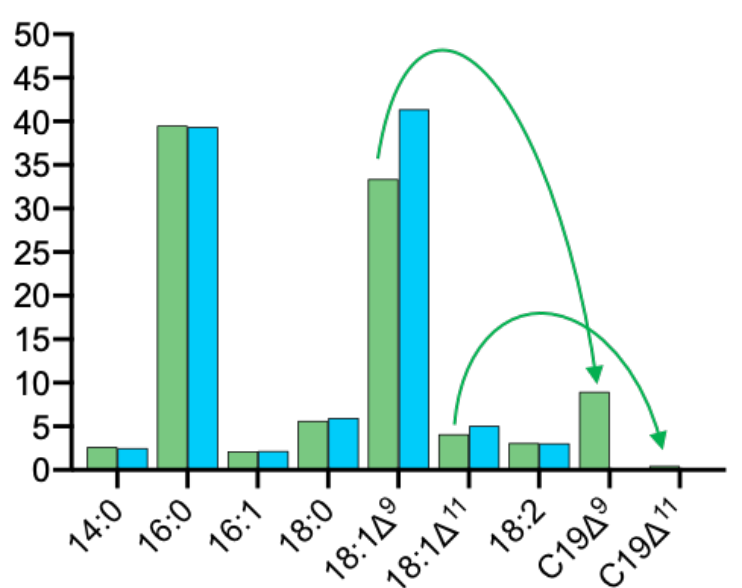

Figure 3. Fatty acid profiles of $E$. faecalis WT, $\Delta$ FASII, and $\Delta c f a$ strains grown without and with serum. E. faecalis was grown in $\mathrm{RPMI}^{+}$or in $\mathrm{RPMI}^{+}$containing $40 \%$ human serum to stationary phase. FA composition was compared to assess the roles of medium FA content and genetic background. The means of the percentages of each FA found in E. faecalis, BWT or mutant strains, grown to stationary phase in the presence or absence of $40 \%$ serum of three independent experiments are presented. (see also Table S2, S3, S4). (A) BWT-FASII grown in the absence or presence of serum. (B). BWT-FASII and Ef $\triangle$ FASII strains grown in $40 \%$ serum. (C) BWT-cfa and Ef $\Delta$ cfa strains grown in RPMI ${ }^{+}$. (D) BWT-cfa and Ef $\Delta$ cfa strains grown in $40 \%$ human serum. Only major fatty acids ( $>2 \%$ of total in at least one profile) are shown. Green stars, fatty acids derived from serum. Green arrows: cyclopropanation in the BWT strains of unsaturated fatty acids, dark green, produced by E. faecalis (mainly 18:1 $1 \Delta^{11}$ ) or, light green, incorporated from serum (mainly $18: 1 \Delta^{9}$ ). Columns: dark green BWT-FASII; dark blue, Ef $\Delta$ FASII; light green, BWT-cfa; light blue, Ef $\Delta$ cfa; hatched, strains grown in $\mathrm{RPMI}^{+}$; empty, strains in $\mathrm{RPMI}^{+}$supplemented with 
A
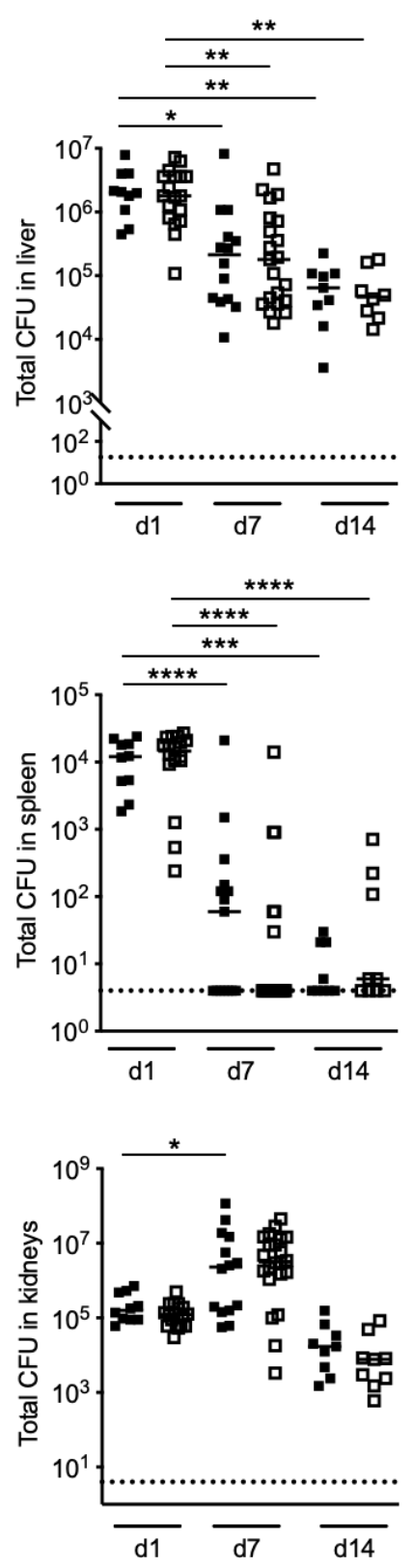

B
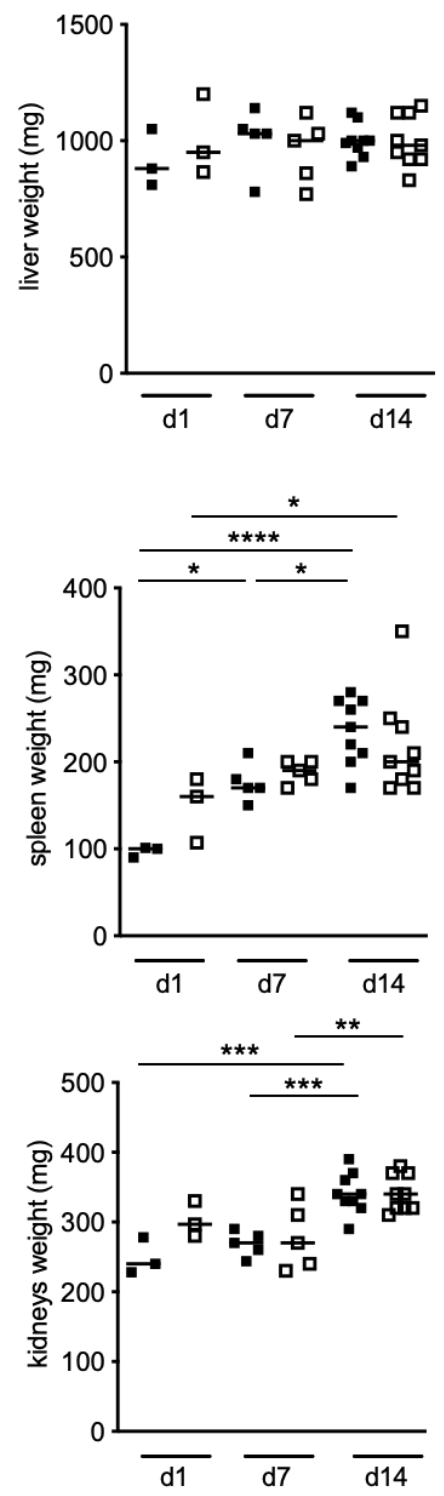

C
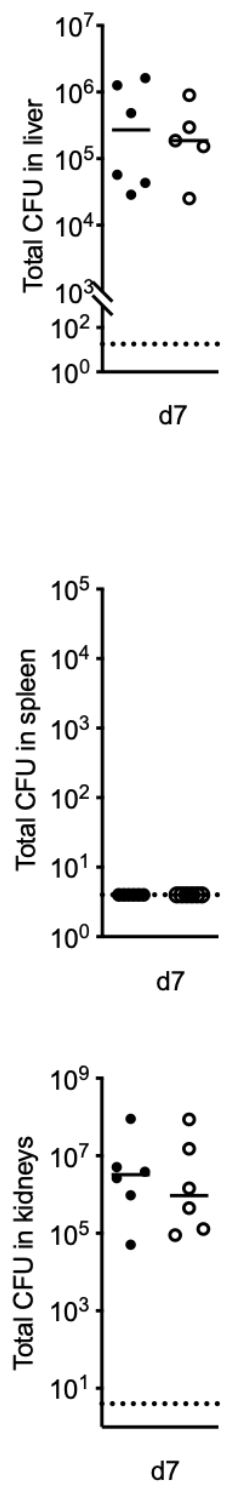

Figure 4. EfAFASII and BWT-FASII infection in a mouse septicemic model. (A) CFUs shown by scattergraph plots at days 1, 7 and 14 pi of bacteria recovered from BWT-FASII and Ef $\Delta$ FASII infected mice. Nine to 20 mice were intravenously infected with $6.10^{8}$ to $8.10^{8} \mathrm{E}$. faecalis BWTFASII (closed squares) or Ef $\triangle$ FASII (open squares). (B) Organ weights shown by scattergraph plots at days 1, 7 and 14 pi of 3 to 9 mice infected by Ef $\Delta$ FASII or BWT-FASII. Symbols are as in 'A'. (C) CFUs shown by scattergraph plots at day 7 pi of viable bacteria recovered Ef $\Delta \mathrm{cfa}$ and BWT-cfa infected mice. Six mice were intravenously infected with 6-8.10 ${ }^{8}$ E. faecalis BWT-cfa (closed circles) or Ef $\Delta$ cfa (open circles) strains. Upper panels, liver; middle panels, spleen; lower panels, kidneys. Each symbol represents the number of CFUs per organ of one infected mouse or the weight of one organ. Median ranges are represented. Statistical analysis, A, B, 2-way ANOVA and a Bonferroni post-test; C, Mann-Whitney test. * $\mathrm{p}<0.05$; ** $\mathrm{p}<0.01$, *** $\mathrm{p}<0.005 * * * *$ $542 \mathrm{p}<0.0001$. 